\title{
Archéopages Archéopages
}

Archéologie et société

Hors-série 1 | 2008

Constructions $^{s}$ de l'archéologie

\section{L'ethnoarchéologie : de la ferme de La Tour au tell de Houlouf, en passant par un rêve brésilien}

Augustin F. C. Holl

\section{(2) OpenEdition}

1 Journals

Édition électronique

URL : https://journals.openedition.org/archeopages/861

DOI : 10.4000/archeopages.861

ISSN : 2269-9872

Éditeur

INRAP - Institut national de recherches archéologiques préventives

Édition imprimée

Date de publication : 1 février 2008

Pagination : 79-84

ISSN : 1622-8545

\section{Référence électronique}

Augustin F. C. Holl, «L'ethnoarchéologie : de la ferme de La Tour au tell de Houlouf, en passant par un rêve brésilien », Archéopages [En ligne], Hors-série 1 | 2008, mis en ligne le 01 février 2008, consulté le 24 février 2023. URL : http://journals.openedition.org/archeopages/861 ; DOI : https://doi.org/ 10.4000/archeopages.861 
de rapports de parenté matrilinéaires au sein d'une société disparue à partir de la présence de traces de céréales incrustées dans des poteries qu'on supposait avoir été fabriquées par les femmes. Le raisonnement était difficile à suivre et les preuves inexistantes.

Enfin, je voudrais ajouter que les archéologues ont augmenté notre connaissance en nous faisant découvrir de multiples sites au sein desquels des sociétés célébraient leurs dieux ou communiquaient avec les esprits de la nature qui les entourait. L'existence et la multiplicité de ces sites posent la question des rapports changeants entre le divin et l'humain, ainsi que le problème de l'importance des rites pour agir sur la nature et pour établir et conserver un ordre social. Or l'une de nos grandes difficultés, tant pour les archéologues que pour les ethnologues occidentaux, est de comprendre la rupture qu'a représentée l'apparition, au Proche-Orient, de religions monothéistes. La plupart des sociétés humaines ont été, et pour beaucoup d'entre elles continuent d'être, polythéistes. Or le rapport au divin et au sacré est totalement différent pour ceux qui croient en une religion polythéiste. Les hommes ont alors le sentiment d'accomplir les rites avec leurs dieux et leurs ancêtres. Ceux-ci sont présents à leur côté lorsqu'ils célèbrent les rites ou se rendent visibles en s'incarnant, par exemple, provisoirement dans le masque des danseurs.

N'oublions pas que - pour évoquer ici l'Europe préchrétienne-, dans la Rome antique, toutes les grandes cérémonies comportaient des sacrifices. Ces sacrifices se concluaient par un banquet où les dieux des Romains étaient assis aux côtés des magistrats et des prêtres qui avaient célébré les rites. La distance entre l'humain et le divin était donc totalement différente de celle introduite par le monothéisme. L'idée qu'il n'existe qu'un seul Dieu - qui a tout créé à partir de rien implique et impose l'idée d'une distance infinie entre les hommes et Dieu, d'une transcendance divine irréductible. De ce fait, les hommes ne peuvent vivre leurs rapports à Dieu que dans un état de subordination absolue. Leur destin dépend de sa grâce et, de ce fait, logiquement de l'amour que Dieu peut avoir pour les hommes. Or aimer les hommes n'était pas une caractéristique obligée ni attendue des dieux des religions polythéistes: les sacrifices qu'on leur rendait n'avaient pas le même sens que les prières et les rites des religions monothéistes. Quelles traces ont donc laissé pour nos générations et pour les archéologues qui les analysent aujourd'hui tous ces domaines de la vie sociale et toutes les différences inventées par les hommes dans la manière d'organiser cette vie et de se représenter l'univers? Une conclusion s'impose: archéologues, ethnologues, historiens ne peuvent que travailler ensemble pour reconstituer avec un peu de «vrai-semblance » quelques morceaux de notre histoire. Aucune de ces disciplines n'y suffira jamais à elle seule, et c'est mieux de le savoir.

\section{L'ethnoarchéologie: de la ferme de La Tour au tell de Houlouf, en passant par un rêve brésilien}

\author{
Augustin F. C. Holl \\ Museum of Anthropology, University of Michigan
}

J uin 1980 : dans la cour de la ferme de La Tour à Paissy, dans l'Aisne, la campagne de fouille archéologique du Centre de recherches protohistoriques de l'université Paris I bat son plein. Cette année-là, je participais pour la première fois à une fouille. Il va sans dire que mes compétences étaient abstraites. J'ai été adopté par l'équipe de la vallée de l'Aisne, et c'est elle qui m’a formé.

Au fil du temps, je me suis intéressé sérieusement aux recherches sur les premières communautés agricoles du Bassin parisien. Les sites de Cuiry-lès-Chaudardes et Berry-au-Bac révélaient des groupes de longues maisons, caractéristiques de ces communautés pionnières [Fig.1]. Les membres de l'équipe de la vallée de l'Aisne, ainsi que d'autres chercheurs allemands, belges, néerlandais, polonais, autrichiens, tchécoslovaques, etc., s'attelaient à l'étude des longues maisons néolithiques dans toutes leurs manifestations archéologiques. Chaque maison avait abrité des individus faisant partie d'un groupe social qui se distinguait des autres, tout en formant entre elles une entité sociale globale, robuste, flexible et durable. Quelle était l'éventuelle structure de cette entité sociale? Comment se reproduisait-elle? Comment s'ajustait-elle aux changements démographiques et économiques? Il est difficile, pour l'archéologue, de répondre à ces questions et la tentation était très forte de ne même pas les prendre en considération. Les données archéologiques ne permettaient au mieux que de faire des suggestions. Que faire?

Eurêka! Par la suite et au cours des multiples conversations professionnelles et amicales que j'ai pu avoir durant de mes années parisiennes, j'ai eu l'idée d'une recherche ethnoarchéologique sur la dynamique des maisons longues. Il fallait trouver une région du monde hébergeant encore des sociétés utilisant ce type de maison. Je m'en suis ouvert à un collègue ethnologue nanterrois qui avait alors un projet de recherche au Brésil: il y avait effectivement des sociétés à maisons longues le long du rio Xingu, un important affluent de l'Amazone. Ne pouvant plus aller sur ce terrainlà, il me proposa de me mettre en contact avec un ethnologue de l'Orstom qui travaillait dans cette région du monde, au cours des années 1980. L'éventualité d'un projet ethnoarchéologique en Amazonie m'a alors fait perdre tout sens de la mesure: j'ai oublié que j'avais des cours à donner, un projet archéologique au Nord Cameroun et un autre dans le Néguev en Israël. Le rêve s'est heurté à la dure réalité, 


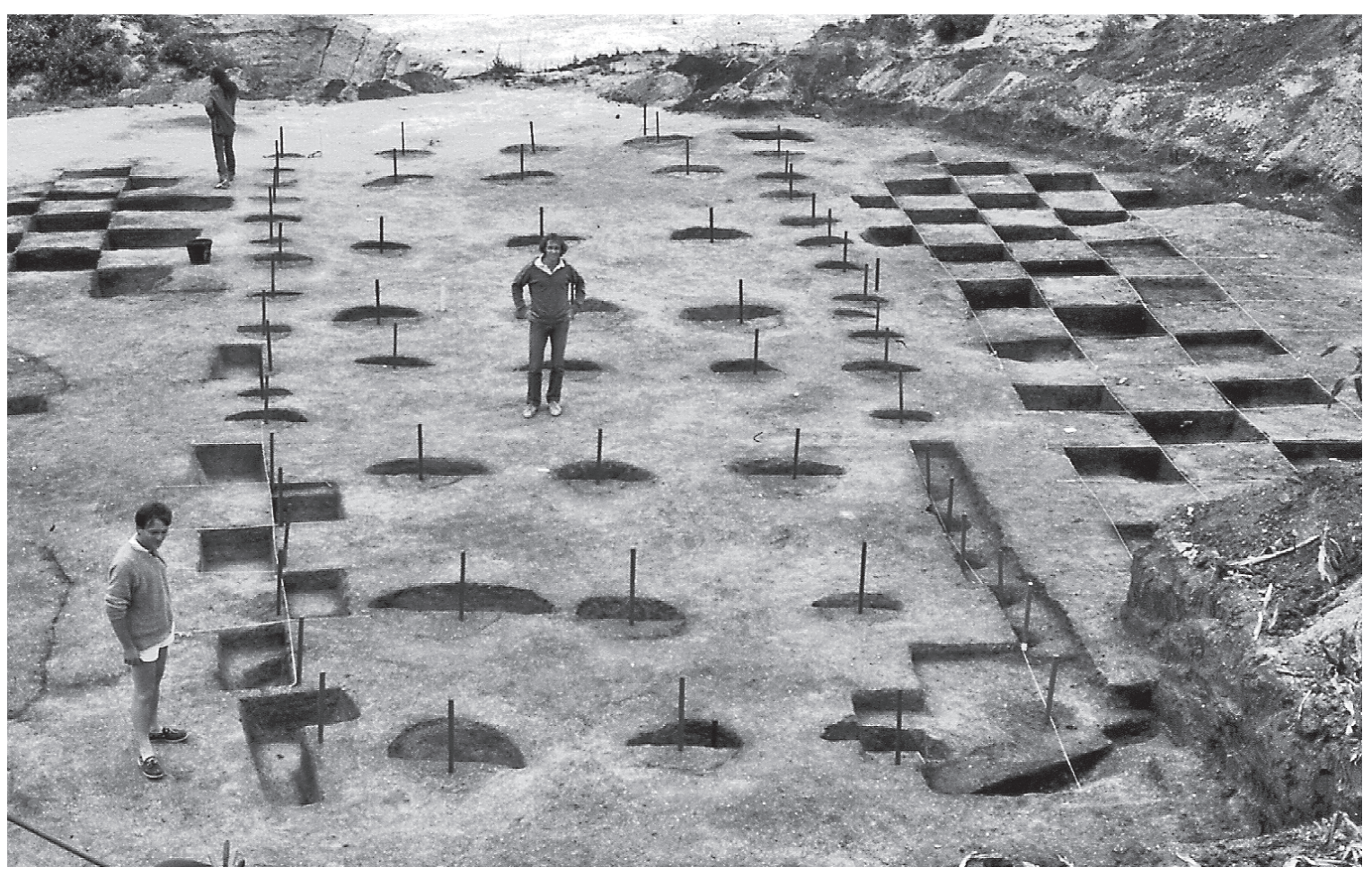

๕

[Fig. 1] Fouille d'une maison longue

du Néolithique rubané dans la vallée

de l'Aisne, à Berry-au-Bac,

Le Chemin de la Pêcherie. 
mais je pouvais encore imaginer que ce n'était que partie remise...

Que fallait-il faire pour comprendre la dynamique sociale et démographique des maisons longues? En théorie, la recherche pouvait s'effectuer à trois niveaux: l'unité d'habitation; le groupe de maisons longues d'une même localité; la répartition régionale des localités à maisons longues. L'analyse de cet habitat brésilien comporterait un relevé cartographique précis, avec des photographies détaillées montrant le nombre de foyers, la répartition des hamacs, la structure de l'espace interne et son rapport à l'espace externe, le nombre d'individus et la composition démograp hique de l'unité domestique, le mode de recrutement des membres, etc. Létude du «village» inclurait elle aussi un relevé cartographique précis de l'habitat, l'analyse du voisinage, l'espacement entre les unités résidentielles, le recensement de la population, ainsi que les diverses formes de variation entre maisons longues (dimension, structure interne, nombre d'habitants, etc.). Létude régionale, quant à elle, mettrait l'accent sur le système d'implantation des villages, l'examen des variations entre villages pour ce qui est de la population, du nombre de maisons longues, des rapports d'alliance, de neutralité ou d'antagonisme. L'idée d'entreprendre des recherches dans des sociétés contemporaines en vue d'éclairer des situations passées n'était pas nouvelle (elle avait, en particulier, été précédée par un long usage de l'analogie ethnographique, plus ou moins directe selon les circonstances), mais elle était, dans la France d'alors, assez originale.

La notion d'ethnoarchéologie. Les limites des monographies ethnologiques ont contraint certains archéologues à faire du terrain ethnographique à des fins archéologiques. L'ethnoarchéologie est dans cette perspective une discipline de terrain. Il n'est pas excessif de dire qu'il existe autant d'ethnoarchéologies que d'ethnoarchéologues.

Selon Clark (1968, p. 280), «l'opération [...] procède en deux temps: dans un premier temps, faire du terrain pour collecter des données ethnoarchéologiques et, dans un second temps, appliquer celles-ci aux situations archéologiques avec le maximum de précision de manière à s'assurer la reconstitution la plus plausible ». Pour Ian Hodder (1982, p. 12), « chaque aspect de la culture matérielle - que ce soit une tombe, l'habitat, la structure des murs ou la répartition des détritus - peut être interprété en termes de schèmes communs. Ces structures de significations imprègnent tous les aspects des données archéologiques. Chaque élément matériel est significatif en vertu de sa place dans l'ensemble ». ${ }^{2}$ Ces deux définitions se situent aux extrêmes d'un même continuum. Dans le premier cas, l'ethnoarchéologie n'a de justification que par rapport aux problèmes archéologiques auxquels elle est censée fournir une solution.
Dans le second cas, l'étude de la culture matérielle est, en soi, la véritable raison d'être de l'ethnoarchéologie. Point n'est besoin de choisir entre ces deux positions, toutes valides et légitimes. Les problématiques ethnoarchéologiques se sont déployées dans toutes les directions (Audouze 1992 ; David, Cramer 2001) et dans plusieurs parties du monde. Les recherches se sont par ailleurs développées en taphonomie et analyse de la formation et de la perturbation des sites archéologiques, avec un accent particulier sur les campements de chasseurs collecteurs. L'hypothèse du charognage a provoqué une flambée de recherches sur l'accès aux carcasses animales avec analyse systématique de la succession des signatures carnivore/charognard. Les structures d'habitat, la technologie, la production des outils en pierre, de la poterie, des objets métalliques, les échanges, l'identité ethnique et l'analyse du style... tous ces éléments ont fait l'objet d'observations ethnoarchéologiques plus ou moins systématiques.

En 1982, Ian Hodder publiait Symbols in Action: Ethnoarchaeological Studies of Material Culture. J'ai passé une nuit blanche à lire ce passionnant ouvrage sur les potentialités des recherches ethnoarchéologiques sur la culture matérielle. Les chapitres ambitieux s'y succédaient sur des thèmes à forte implication archéologique. L'approche contextuelle qu'Ian Hodder (1982, p. 218) développait à la fin de l'ouvrage m'avait alors plutôt séduit, et je m’étais particulièrement intéressé à l'habitat nuba. Avec le temps, cependant, il m'est apparu de plus en plus difficile de reconstituer les séquences méthodologiques en partant du présupposé de la lecture de la «culture matérielle comme texte» pour aller vers l'interprétation de la symbolique du rejet des détritus chez les Nuba. N'y avait-il pas là un cercle vicieux? Comment développer un modèle de recherche ethnoarchéologique dans lequel les questions ne présupposaient pas les réponses?

L'ethnoarchéologie de l'habitat arabe shuwa. Les Arabes Shuwa se sont installés dans la région de Houlouf probablement au cours du XVII ${ }^{\mathrm{e}}$ siècle, au terme de longs et complexes mouvements migratoires qui ont conduit leurs ancêtres de la vallée du Nil dans la zone du lac Tchad. Leur présence est signalée, au Kanem oriental, en 1391-1392 dans une lettre du roi du Kanem d'alors au sultan Al-Malik al-Zahir Abu Sa'id Barquq d'Égypte (Al-Qalqashandi, in Levtzion and Hopkins 1981, p. 347). Ce sont avant tout des communautés de pasteurs (élevant bovins et caprinés) qui se sont infiltrés dans la plaine tchadienne. Les recherches ethnoarchéologiques sur l'habitat arabe shuwa du Nord Cameroun n'ont pas été planifiées en tant que telles. En 1982, le programme de travail de la mission archéologique du Nord Cameroun était essentiellement archéologique, s'articulant sur la fouille du tell de Houlouf (Holl 2002). Chaque année, cependant, une partie de la région 

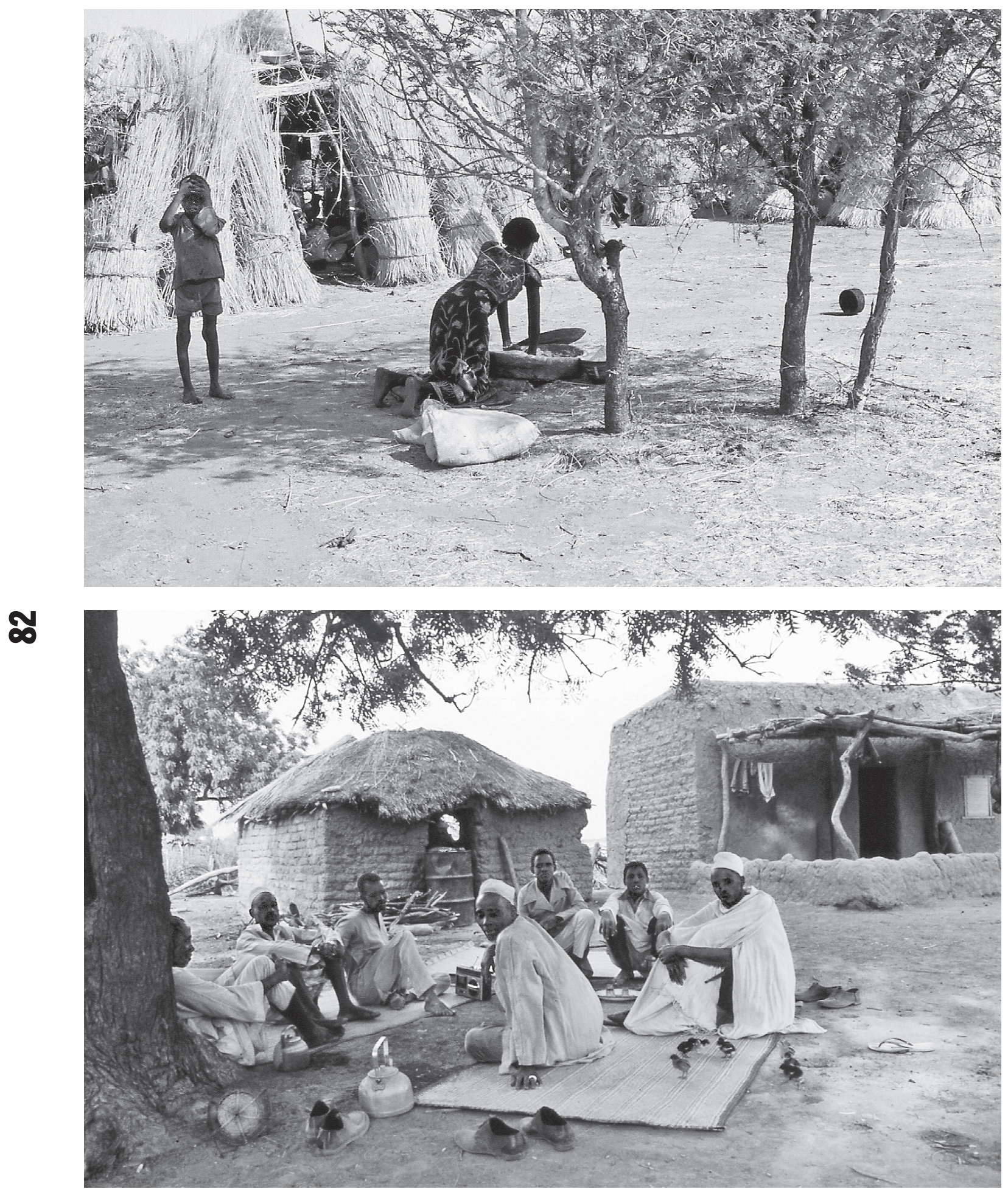

[Fig. 2] L'un des trois types d'habitat identifiés chez les Arabes Shuwa: le camp de saison sèche.
[Fig.3] L'habitat dans le village de Djidat, à droite du cliché, montre un bâtiment rectangulaire avec un toit en terrasse. Entouré de champs, il est typique de l'habitat sédentaire. 
de Houlouf faisait l'objet d'une prospection intensive; c'est au cours de ces prospections que nous avons observé des villages abandonnés, avec un impressionnant répertoire de la culture matérielle. L'idée d'entreprendre une étude systématique de ces villages s'est rapidement imposée et, en trois campagnes, nous avons collecté des données sur les multiples facettes de l'habitat arabe shuwa de la région de Houlouf (Holl 2004). La méthode de terrain était strictement archéologique. L'intention explicite était d'étudier les processus de formation des sites archéologiques, puis d'aborder les questions relatives à l'implantation régionale de l'habitat arabe shuwa par rapport à celui des Kotoko, en comparant la structure des villages (enclos à bétail, unités domestiques, éléments de la culture matérielle, etc.).

L'habitat arabe shuwa se compose de trois principales variantes: des villages de groupes sédentarisés, des villages semi-permanents de groupes semi-nomades, et des camps de saison sèche utilisés par tous [Fig.2]. Le village arabe shuwa est de forme circulaire ou ovale, les habitations pour humains et animaux entourant l'espace central des enclos à bestiaux. L'implantation régionale et la forme des villages ne font sens que si l'on comprend l'histoire des relations ambiguës et généralement tendues entre Kotoko et immigrants arabes shuwa.

Ce travail a abouti à des résultats inattendus. De fait, cette recherche ethnoarchéologique a jeté un éclairage intéressant sur l'histoire mouvementée de la région, les relations ambiguës entre les Arabes Shuwa et leurs suzerains kotoko; et finalement (en raison d'une dynamique démographique plus soutenue) sur l'émergence d'une "puissance » arabo-shuwa locale qui a supplanté la traditionnelle chefferie kotoko de Houlouf. Les détails architecturaux, l'agencement des bâtiments et les structures de l'organisation spatiale des villages étudiés apparaissaient clairement comme de véritables indicateurs socioéconomiques. Les bâtiments rectangulaires avec toit en terrasse et les anneaux concentriques de champs dans un rayon de l'ordre de 2,5 km étaient l'exclusivité des communautés sédentaires [Fig.3]. L'habitation circulaire avec toit conique en chaume était la norme des villages de pasteurs semi-nomades. L'habitation des campements de saison sèche était, quant à elle, en forme de dôme. En somme, la sédentarisation se marquait par des changements architecturaux, avec l'adoption progressive de maisons rectangulaires en brique avec toiture en terrasse un des traits bien connus des archéologues travaillant sur les changements marquant la transition entre Mésolithique-Épipaléolithique et Néolithique ancien.

L'ethnoarchéologie comme générateur d'hypothèses. Les données collectées dans le programme ethnoarchéologique de la région de Houlouf ont aidé à formuler de nouvelles hypothèses nuancées sur la genèse des communautés israélites dans le Levant méridional entre -1200 et -900 (Levy, Holl 2002). En s'appuyant sur la réussite des Arabes Shuwa dans la région de Houlouf, il est apparu que les multiples hypothèses sur la genèse des Israélites pouvaient être évaluées de manière plus parcimonieuse. Les modèles de conquête triomphale - les trompettes de Jéricho et de symbiose graduelle semblent être les moins plausibles. L'ethnogenèse des Israélites semble découler de processus migratoires, préludes à des infiltrations pacifiques, culminant avec la prise de contrôle des centres de pouvoir régionaux. Ce modèle ancré sur l'Exode s'articule en trois temps : l'expulsion d'Égypte, les quarante ans d'errance dans le désert du Sinaï, et la prise de contrôle de la région par les Israélites. Le processus d'émergence des Israélites, leur différentiation des Shasu (groupe générique de pasteurs nomades de Transjordanie Moab et Edon) dérivent d'un contraste radical avec les Égyptiens, pour ce qui est des manières, des coutumes et des pratiques cultuelles. Ces antagonismes se seraient cristallisés à la fin de l'âge du Bronze final et au premier âge du Fer, dans le contexte de l'expansion philistine et le renouveau de l'impérialisme égyptien au cours des $\mathrm{XII}^{\mathrm{e}}$ et $\mathrm{XI}^{\mathrm{e}}$ siècles avant notre ère. Des groupes de Canaanites pris entre les puissances combattantes abandonnèrent le littoral méditerranéen pour migrer vers les collines de Galilée, où ils s'insérèrent dans une mosaïque de communautés aux origines diverses.

Ces multiples groupes s'agrégèrent en un certain nombre d'unités tribales, qui, au cours du second âge du Fer, développèrent un État territorial et un système monarchique. Les plans de sites des collines de Galilée attestent un arrière-plan pasteur-nomade dans la forme et la juxtaposition des structures fouillées (Finkelstein 1988).

La traduction archéologique. La recherche ethnoarchéologique produit des formes complexes d'analogies, certaines ponctuelles, d'autres relationnelles. Les deux options sont importantes pour une interprétation robuste et flexible des données archéologiques. Les principes de transfert des résultats ethnoarchéologiques vers les situations archéologiques et les termes de cette traduction dérivent de deux types de considérations. Le principe uniformitariste selon lequel les mêmes processus engendrent les mêmes résultats - justifie le transfert indépendamment de l'espace et du temps. Le principe de «continuité historique» ouvre la possibilité d'utilisation des observations actuelles pour interpréter les données archéologiques dans les cas de «descendance culturelle » directe. Chacune des deux options présente des avantages considérables, mais aussi des limites importantes. Dans tous les cas, il est nécessaire d'ajuster les échelles d'observation, comme le montrent les résultats du projet ethnoarchéologique de l'habitat arabe shuwa, qui permettent d'éliminer les modèles les moins 
pertinents pour expliquer la genèse des communautés israélites des collines de Galilée, au cours des $\mathrm{II}^{\mathrm{e}}$ et $\mathrm{I}^{\mathrm{er}}$ millénaires avant notre ère. Dans un premier temps, il y a eu acte de violence étatique, les Arabes Shuwa étant les descendants des pasteurs nomades arabes expulsés de la vallée du Nil par les armées mameloukes, les Israélites étant les descendants des expulsés d'Égypte. Puis est apparue une phase migratoire plus ou moins longue, avec un mouvement vers le lac Tchad dans un cas, et des pérégrinations dans le désert du Sinaï dans l'autre. On observe ensuite une infiltration de petits groupes aux destins divers qui développèrent un sens de collectivité de destin face à l'adversité. Enfin, il y eut prise de pouvoir local. Les données archéologiques sous forme de carte d'implantation des sites, plans des structures d'habitat et des enclos à bétail ont alors été utilisées pour choisir les hypothèses les plus plausibles.

Conclusion. L'espace limité de cet article ne permet pas une analyse plus nuancée de l'évolution de l'ethnoarchéologie. Celle-ci est passée de la pratique des « discours édifiants» portant sur les limitations des interprétations archéologiques à des procédures de terrain ambitieuses, impliquant des programmes de longue durée. De fait, l'ethnoarchéologie - en mettant l'accent sur l'usage et la manipulation de la culture matérielle - a enrichi les pratiques archéologiques d'une puissante source d'inspiration.

Audouze F. (dir.) 1992 : Ethnoarchéologie: Justification, problèmes, Limites, Rencontres internationales d'archéologie et d'histoire d'Antibes, Juan-les-Pins, Éditions ApDCA.

ClARK J. D. 1968: «Studies of Hunter-gatherers as an aid to the interpretation of prehistoric societies", in R. B. Lee and I. DeVore (dir.), Man the Hunter, Chicago, Aldine, p. 276-28o.

David N., Kramer C. 2001: Ethnoarchaeology in Action, Cambridge, Cambridge University Press.

FinKelstein I. 1988: The Archaeology of Israelite Settlement, Jerusalem, Israel Exploartion Society.

Hodder I. 1982: Symbols in Action, Cambridge, Cambridge University Press.

Holl A. F. C. 2002: The Land of Houlouf: Genesis of a Chadic Chiefdom (BC190o-AD180o), Ann Arbor, The University of Michigan Museum of Anthropology.

Holl A. F. C. 2004 : Ethnoarchaeology of Shuwa-arab Settlements, Lanham, Lexington Books.

Levtzion N., Hopkins J. F. P. (dir.) 1981: Corpus of Early Arabic Sources for West African History, Cambridge, Cambridge University Press

LEVY E. L., Holl A. F. C. 2002 : «Migrations, Ethnogenesis, and Settlement Dynamics: Israelites in Iron Age Canaan and ShuwaArab in the Chad Basin ", Journal of Anthropological Archaeology, $\mathrm{n}^{\circ} 21, \mathrm{p} .83-118$

\section{Archéologie et diversité culturelle}

\author{
Luc Jallot \\ Inrap, UMR 5140, Archéologie des sociétés méditerranéennes, \\ milieu, territoire, civilisations
}

C'est en opposant les singularités qu'on comprend la richesse de l'universel. L'humanité n'est pas une fourmilière mais l'expression d'une diversité... Le fait d'être de quelque part donne conscience que chaque homme est un centre du monde. Félix Marcel Castan
Ainsi je suis responsable pour moi-même et pour tous, et je crée une certaine image de l'homme que je choisis; en me choisissant, je choisis l'homme. Jean-Paul Sartre

E ntre la fuite et la quête. Le mot «diversité » se rapporte à des domaines aussi différents que l'origine ethnique, la religion,

la culture, l'orientation sexuelle, les capacités physiques et mentales, les espèces animales et végétales. À son opposé, on trouve des termes comme « unitarisme», «nivellement», "protectionnisme», «standardisation du mode de vie », «banalisation des espèces». Cette opposition est ambiguë: l'humanité n'a jamais semblé aussi variée et la nature autant diversifiée. Jamais nous n'avons eu autant conscience de la fragilité de cette diversité. Jamais la typicité de chaque société et l'unicité de chaque être humain n'ont été autant affirmées. L'immunologie nous certifie le caractère absolument singulier de notre organisme, la biologie nous explique que notre code génétique est le nôtre et celui de personne d'autre, même les religions insistent sur notre relation particulière ou intime avec les forces surnaturelles, et davantage encore, notre cerveau demeure sans équivalent car l'individuation de chaque personnalité est un processus qui ne s'arrête qu'avec la mort. Aucun de nous ne peut donc penser comme un autre. L'uniformisation grandissante du monde tient, quant à elle, à autre chose. Ne sont-ce pas les aspirations humaines qui se ressemblent? Tous semblables parce que tous égaux ou du moins culturellement équivalents ou tous nivelés, invisibles, parce que la communication - ce bruissement intense qui tisse la trame du monde - brouille les pistes et apporte l'illusion du dialogue, quand les pays riches se barricadent dans leurs frontières et que s'établissent des traités de paix commerciale dictés par les économies les plus fortes? Les désenchantements sont à la mesure de cette solitude où se lisent la perte de l'altérité et la quête d'une «diversité culturelle».

\section{Patrimoine archéologique, patrimoine} orphelin. La préservation des sociétés humaines, traditionnelles notamment, et de leur histoire est indissociable d'une diversité qu'elles maintiennent, réduisent ou accroissent. Ainsi, la déclaration, accompagnée d'un plan d'application, adoptée par l'Unesco en 2002 à Johannesbourg, souligne que «la diversité culturelle et la diversité biologique ont la même signification et la même importance pour le développement durable ». D'autres pétitions internationales proposent une définition d'un patrimoine culturel immatériel ou patrimoine vivant comme « creuset de la diversité culturelle et garant du développement durable» (Unesco 2003). Par ailleurs, plusieurs conventions émanent du Conseil de l'Europe et permettent d'associer patrimoine culturel et patrimoine archéologique (en particulier, celles de 1955 à Paris et à Grenade, et celle de 2005 à Faro). ${ }^{2}$ Les sites archéologiques sont considérés 